\title{
Medications for opioid use disorders: clinical and pharmacological considerations
}

\author{
Nora D. Volkow and Carlos Blanco \\ National Institute on Drug Abuse, Bethesda, Maryland, USA.
}

$T_{1}$ he opioid epidemic, now in its second decade, is one of the most challenging public health crises in the US. Providing an effective response is complicated by multiple underlying causes and consequences as well as a misunderstanding of addiction and the medications used to treat it (1). Indeed, medications for opioid use disorder (MOUDs) are the most effective interventions for treating opioid addiction, but are not prescribed to many who would benefit. Here, we describe the distinction between physical dependence and addiction along with its implication for treatment, and discuss the mechanisms of action of MOUDs.

\section{Opioid withdrawal versus opioid dependence}

Opioid use disorder (OUD) is defined as a pattern of maladaptive opioid use that leads to significant impairment or distress. Severity is classified on the basis of the number of symptoms (Table 1) present: mild (one or two), moderate (three or four), and severe (six or more) (2). In this description, opioid addiction corresponds to moderate and severe OUD.

In diagnosing OUD, many confuse opioid physical dependence with OUD, yet this distinction is crucial for selecting treatment. Physical dependence develops rapidly and occurs in most people who are given repeated doses of opioid medications and manifests as the emergence of acute withdrawal symptoms following discontinuation of opioid drugs. When physical dependence is associated with tolerance, it can lead to a diagnosis of mild OUD (two of the criteria in Table 1). Note that physical dependence and tolerance will be present in many pain patients who are properly treated with opioid medications; hence, the distinction from mild OUD requires the clinician to assess whether significant impairment or distress is present. Physical dependence and the associated acute withdrawal symptoms are adaptations that recover rapidly (within days) and can be managed by slowly tapering the opioid drug without the need of maintenance on opioid medications (3). In contrast, opioid addiction develops in less than $10 \%$ of those exposed repeatedly to opioids and is the result of neuroplastic adaptations in brain circuits underlying reward and motivation, self-regulation and decision-making, and mood and stress reactivity that are long lasting, persisting years after drug discontinuation $(4,5)$. Opioid addiction significantly benefits from the use of medications for OUD.

Abrupt cessation of opioids after repeated use can produce an intense but rarely life-threatening withdrawal syndrome, which can be understood as an adaptation to maintaining homeostasis or allostatic process (3). Common symptoms of early withdrawal include mydriasis, piloerection, muscle twitching, lacrimation, rhinorrhea, diaphoresis, yawning, tremor, insomnia, restlessness, myalgia, arthralgia, diarrhea, and nausea or vomiting. As withdrawal progresses, tachycardia, tachypnea, hypertension or hypotension, and dehydration can appear. Note that this is distinct from the protracted withdrawal syndrome characterized by dysphoria, craving, and insomnia that reflects brain circuitry neuroadaptations associated with addiction. Symptoms of acute withdrawal (as well as protracted withdrawal) can be a powerful trigger for relapse for individuals with OUD (1), but can also lead to opioid seeking in pain patients in whom acute opioid withdrawal is not properly managed.

Multiple neuroadaptations underlie physical dependence (6), including

Conflict of interest: The authors have declared that no conflict of interest exists.

Copyright: ( 2020 , American Society for Clinical Investigation.

Reference information: / Clin Invest. 2020;130(1):10-13. https://doi.org/10.1172/JCI134708.

desensitization and internalization of the $\mu$-opioid receptor (MOR), impaired MOR signaling with intracellular effectors, intracellular upregulation of cAMP/PKA in opioid-sensitive neurons, adaptations in neuropeptide systems that interact with $\mu$-opioid-sensitive neurons, and activation of glial signaling (7). Hyperactivity of the locus coeruleus (LC) underlies many of the symptoms of acute withdrawal, and $\alpha_{1}$ adrenergic agonists, such as lofexidine and clonidine, which reduce noradrenergic release, are useful for the management of acute opioid withdrawal.

In contrast to withdrawal, which is a physiological response to the abrupt decline in MOR occupancy and signaling, addiction is predominantly a disorder of brain circuits that impairs motivation, self regulation, and hedonic tone. The brain mechanisms underlying addiction include the following (3): (a) reward circuitry, originating in the dopamine neurons in the ventral tegmental area and projecting to the nucleus accumbens, ventral prefrontal cortex, and amygdala; (b) emotional circuitry, including the hippocampus, extended amygdala, lateral habenula, dorsal raphe, and insula; and (c) executive control circuitry, which involves widely distributed and complex prefrontal cortex-subcortical circuitry (3). In addition, circuitry involved in interoception modulates awareness of drug-conditioned cues, stress, and negative emotional states (3). Disruption of these circuits underlies the compulsive pattern of drug taking despite its adverse consequences (8).

\section{MOUDs}

MOUDs are the standard of care for OUD (9). They are associated with reduced risk of relapse, overdose deaths, infections, and criminal behavior and are more cost-effective than no OUD treatment or treatment with no medication. There are three medications approved by the FDA for the treatment of OUD: metha- 
Table 1. DSM-5 criteria for diagnostic criteria OUD

A. A problematic pattern of opioid use leading to clinically significant impairment or distress, as manifested by at least two of the following, occurring within a 12-month period:

1. Opioids are often taken in larger amounts or over a longer period than was intended.

2. There is a persistent desire or unsuccessful efforts to cut down or control opioid use.

3. A great deal of time is spent in activities necessary to obtain the opioid, use the opioid, or recover from its effects.

4. Craving, or a strong desire or urge to use opioids.

5. Recurrent opioid use resulting in a failure to fulfill major role obligations at work, school, or home.

6. Continued opioid use despite having persistent or recurrent social or interpersonal problems caused or exacerbated by the effects of opioids.

7. Important social, occupational, or recreational activities are given up or reduced because of opioid use.

8. Recurrent opioid use in situations in which it is physically hazardous.

9. Opioid use is continued despite knowledge of having a persistent or recurrent physical or psychological problem that is likely to have been caused or exacerbated by the substance.

10. Tolerance, as defined by either of the following:

a. A need for markedly increased amounts of opioids to achieve intoxication or desired effect.

b. A markedly diminished effect with continued use of the same amount of an opioid.

Note: This criterion is not considered to be met for individuals taking opioid under medical supervision.

11. Withdrawal, as manifested by either of the following:

a. The characteristic withdrawal syndrome for opioid.

b. Opioids (or a closely related substance) are taken to relieve or avoid withdrawal symptoms.

Reprinted with permission from the American Psychiatric Association (2).

done (full MOR agonist), buprenorphine (partial MOR agonist, $\kappa$-opioid receptor [KOR] antagonist, and nociceptor receptor agonist), and naltrexone (MOR and KOR antagonist). The MOR is both the therapeutic target for MOUDs and the target for heroin and other opioids when misused for their rewarding effects. For this reason, many have dismissed agonist medications (methadone and buprenorphine) as only substituting one drug for another; however, this view ignores fundamental differences between drugs of abuse and MOUDs. These distinctions include differences in pharmacokinetics, pharmacological effects at the MOR, and doses and routes of administration.

Pharmacokinetics and route of administration. The rate at which opioid drugs enter the brain and bind to the MOR modulates their rewarding effects such that drugs with fast uptake into the brain and that interact rapidly with the MOR, such as heroin and fentanyl, are the most rewarding. The route of administration also affects pharmacokinetics; intravenous or smoking administration results in faster brain delivery than taking orally. When used therapeutically, MOUDs are either given orally or in slow-release formulations, which slows the rate of brain entry and clearance. The relatively slow brain clearance of buprenorphine and, to a lesser extent, methadone leads to milder severity of withdrawal upon their discontinuation than when discontinuing heroin or fentanyl. In this respect, buprenorphine leads to a milder withdrawal than methadone. Additionally, the slower pharmacokinetics of MOUDs result in more stable levels of MOR occupancy than misuse of opioid drugs for their rewarding effects. Stable occupancy of MOR by MOUDs controls opioid craving and prevents the emergence of acute withdrawal symptoms.

Doses and frequency of administration also differ when opioid drugs are misused for their rewarding effects than when used therapeutically. Methadone is typically injected when it is misused. In the case of buprenorphine, its injection is limited by the combination with the antagonist opioid drug naloxone (Suboxone formulation), which has very poor bioavailability when given sublingually, but if injected, will trigger an acute withdrawal. Nonetheless, there are multiple reports of diversion and misuse of buprenorphine, though it appears that misuse of buprenorphine is mostly to alleviate opiate withdrawal or achieve abstinence from other opioids, particularly when access to this medication is restricted (10).

Though MOR is the target for the rewarding and analgesic effects of opi- oids, the KOR is implicated in the aversive negative emotional states associated with addictions. Preclinical data indicate an upregulation of KOR signaling in animal models of addiction that, when blocked, prevents relapse into drug taking (11). Hence, a priority in addiction treatment has been the development of $\kappa$ antagonist medications. In this respect the KOR antagonist effects of buprenorphine and naltrexone are likely to contribute to their therapeutic effects. Additionally, buprenorphine also binds to the nociceptin receptor where its agonist effects could also contribute to its efficacy in OUD (12).

\section{Which MOUD to use}

Although there are no empirically based predictors for selecting a specific MOUD, expert consensus and qualitative studies suggest that the selection should be based on the patient's response to prior treatment with MOUDs, their level of physical dependence, the presence of coexisting conditions, and the patient's preference (9). Often, the selection is determined by what is available in a given treatment program. Increasingly, however, there is recognition that patients might respond better to a particular MOUD depending on their characteristics and that optimal medication may be different during treatment initiation than stabilization. 
Methadone has been available for more than 50 years and has the largest evidence of efficacy (13). Methadone would be indicated in patients with severe tolerance in whom buprenorphine treatment might trigger withdrawal symptoms. In general, there is overall better retention with methadone than buprenorphine and higher methadone doses up to $100 \mathrm{mg} /$ day are associated with better outcomes than lower doses (14). As a full agonist, methadone has no ceiling effect, which increases risk for overdoses when used at doses above the patient's tolerance or when combined with other central nervous depressants such as alcohol, benzodiazepines, heroin, or other synthetic opioids. Methadone is administered daily in an oral formulation. In the US methadone must be administered in licensed outpatient treatment programs (OTPs), which constitutes an important barrier to treatment to many patients, though it might improve outcomes in individuals who benefit from daily behavioral intervention given in OTPs (1). There is interest in exploring expanded access to methadone, such as office-based or via pharmacies (15), and developing extended release formulations of methadone to improve adherence, minimize diversion, and facilitate use in healthcare or justice settings.

Buprenorphine has been available to treat OUD for almost two decades (13). Buprenorphine is prescribed in medical offices by clinicians who require a waiver to do so. There are currently 102,570 waivered clinicians in the US, though many are not treating OUD patients (16). Buprenorphine requires daily or every other day dosing, and typical doses range between 8 to $24 \mathrm{mg}$, with a recommended target dose of $16 \mathrm{mg}$. Optimal responses to buprenorphine have been obtained in OUD patients with depressive symptoms, which might reflect in part the mood-enhancing effect of KOR antagonists. As a partial MOR agonist, buprenorphine can precipitate acute withdrawal in individuals with OUD who use high doses of heroin or fentanyl or have been maintained on high doses of methadone. In those instances, it might be best to initiate treatment with methadone and, after slowly tapering the dose, continue with buprenorphine. Buprenorphine is less likely to induce respiratory depression than methadone, but it can still be lethal when combined with other central nervous depressant substances (17). Extendedrelease (XR) formulations of buprenorphine were recently developed that include an FDA-approved six-month implant, a oneweek formulation that is being reviewed by the FDA, and one-month formulations of buprenorphine, one of which was already approved by FDA (1). Limited data are available regarding the acceptability and efficacy of these new formulations in OUD.

Naltrexone is a MOR antagonist, but the utility of the immediate release formulation for OUD treatment has been limited by poor adherence. The development of a monthly XR-extended release naltrexone (XR-NTX) formulation significantly improved treatment retention and outcomes (18). Naltrexone is an antagonist drug that triggers acute withdrawal if OUD patients are not detoxified prior to induction. Current recommendations are for patients to be abstinent for one week prior to XR-NTX induction, which constitutes a barrier to induct some patients into treatment. Some protocols have been developed for faster supervised medical withdrawal (formerly known as detoxification), but further research is needed before adoption in routine clinical practice. The KOR antagonist effects could contribute to the mood improvements reported in OUD patients treated with naltrexone.

Presence of cooccurring disorders may be another consideration in MOUD selection. For example, naltrexone is also effective for alcohol dependence so comorbid OUD with alcoholism might benefit uniquely from this medication, whereas the KOR antagonist properties of buprenorphine may offer unique benefits for OUD patients with comorbid depression. For pregnant women, methadone or buprenorphine is recommended, due to insufficient data on safety of naltrexone.

\section{Conclusion}

MOUDs are among the most effective interventions for preventing overdose mortality and improving outcomes in patients with OUD. However, stigmatization and lack of understanding of addiction and the medications used to treat OUD have interfered with their implementation. The increased recognition that MOUDs are crucial for controlling the current opioid crisis highlights the impor- tance of engaging healthcare in the screening and treatment of OUD.

The views and opinions expressed in this report are those of the authors and should not be construed to represent the views of any of the sponsoring organizations or agencies or the US government.

Address correspondence to: Nora D. Volkow or Carlos Blanco, 6001 Executive Boulevard, Bethesda, Maryland 20892, USA. Phone: 301.443.6540; Email: nvolkow@ nida.nih.gov (NDV). Phone:301.443.6504; Email: carlos.blanco2@nih.gov (CB).

1. Blanco C, Volkow ND. Management of opioid use disorder in the USA: present status and future directions. Lancet. 2019;393(10182):1760-1772.

2. American Psychiatric Association. Diagnostic and Statistical Manual of Mental Disorders. 5th ed. Washington, DC, USA: American Psychiatric Publishing; 2013.

3. Koob GF, Volkow ND. Neurobiology of addiction: a neurocircuitry analysis. Lancet Psychiatry. 2016;3(8):760-773.

4. Kalivas PW, Volkow ND. The neural basis of addiction: a pathology of motivation and choice. Am J Psychiatry. 2005;162(8):1403-1413.

5. Pérez de los Cobos J, Batlle F, Casas M. [Proposal for improving the integration of drug dependencies in psychiatric nosology]. Actas Luso Esp Neurol Psiquiatr Cienc Afines. 1996;24(2):63-65.

6. Christie MJ. Cellular neuroadaptations to chronic opioids: tolerance, withdrawal and addiction. Br J Pharmacol. 2008;154(2):384-396.

7. Rich MM, Wenner P. Sensing and expressing homeostatic synaptic plasticity. Trends Neurosci. 2007;30(3):119-125.

8. Volkow ND, Wang GJ, Fowler JS, Tomasi D, Telang F. Addiction: beyond dopamine reward circuitry. Proc Natl Acad Sci U S A. 2011;108(37):15037-15042.

9. Substance Abuse Mental Health Services Administration. Medications for Opioid Use Disorder. Treatment Improvement Protocol (TIP) Series, No. 63. Rockville, Maryland, USA: SAMSHA; 2018.

10. Chilcoat HD, Amick HR, Sherwood MR, Dunn KE. Buprenorphine in the United States: motives for abuse, misuse, and diversion. J Subst Abuse Treat. 2019;104:148-157.

11. Chavkin C, Koob GF. Dynorphin, dysphoria, and dependence: the stress of addiction. Neuropsychopharmacology. 2016;41(1):373-374.

12. Ciccocioppo R, Economidou D, Rimondini R, Sommer W, Massi M, Heilig M. Buprenorphine reduces alcohol drinking through activation of the nociceptin/orphanin FQ-NOP receptor system. Biol Psychiatry. 2007;61(1):4-12.

13. Mattick RP, Breen C, Kimber J, Davoli M. Buprenorphine maintenance versus placebo or methadone maintenance for opioid dependence. Cochrane Database Syst Rev. 2014;(2):CD002207. 
14. Faggiano FV-TF, Versino E, Lemma P. Methadone maintenance at different dosages for opioid dependence. Cochrane Database Syst Rev. 2003;(3):CD002208.

15. Gauthier G, Eibl JK, Marsh DC. Improved treatment-retention for patients receiving methadone dosing within the clinic providing physician and other health services (onsite) versus dosing at community (offsite) pharmacies. Drug Alcohol Depend. 2018;191:1-5.

16. SAMHSA. Practitioner and Program Data. https://www.samhsa.gov/medication-assistedtreatment/training-materials-resources/ practitioner-program-data. SAMHSA website. Accessed November 7, 2019.

17. Lofwall MR, Walsh SL. A review of buprenor- phine diversion and misuse: the current evidence base and experiences from around the world. J Addict Med. 2014;8(5):315-326.

18. Krupitsky E, Nunes EV, Ling W, Illeperuma A, Gastfriend DR, Silverman BL. Injectable extendedrelease naltrexone for opioid dependence: a double-blind, placebo-controlled, multicentre randomised trial. Lancet. 2011;377(9776):1506-1513. 